\title{
THE MAGELLANIC CLOUDS
}

\section{RAYMOND HAYNES AND DOUGLAS MILNE (EDS.)}

The Magellanic Clouds are very interesting in many respects. They are close to our Galaxy and can be studied in great detail. They are different from our Galaxy and offer therefore a large variety of environments and processes not so much seen in our Galaxy. They are the closest galaxies that we can see in their entirety.

New data have been gathered by modern instruments over the past decade. This IAU Symposium presents an up-to-date summary of the research results obtained from observing the Magellanic Clouds. Star formation in the Clouds is of course a very interesting topic covered, but also the evolution of stars and the chemical composition of these galaxies take a large part of this book. Next to these, the kinematics of each Cloud and the dynamical interaction between them are treated extensively. 